\title{
Enhanced tunability and phase transition studies in compositionally varying lead magnesium niobate-lead titanate multilayered thin films
}

\author{
R. Ranjith, Apurba Laha, and S. B. Krupanidhi ${ }^{\text {a) }}$ \\ Materials Research Center, Indian Institute of Science, Bangalore 560012, India
}

\begin{abstract}
Multilayers of $(1-x) \mathrm{Pb}\left(\mathrm{Mg}_{1 / 3} \mathrm{Nb}_{2 / 3}\right) \mathrm{O}_{3}-x \mathrm{PbTiO}_{3}$ thin films were prepared with a compositional variation of $\mathrm{PbTiO}_{3}$ on each layer across the film by pulsed laser ablation technique. The fabricated films were found to possess an enhanced diffusiveness in the dielectric phase transition, which actually seemed to be an envelope of the phase transition of the individual layer relaxor ferroelectrics of a particular composition. They also showed a very high nonlinear dielectric response and hence a high tunability about $70 \%$, an increase of about $40 \%$ than that observed for single layer composition. This increase in tunability has been attributed to the lattice strain and the artificially enhanced chemical heterogeneity of the system. These multilayer films also showed a slim hysteresis loop characteristic with a saturation and remnant polarization of 20 and $6 \mu \mathrm{C} / \mathrm{cm}^{2}$, respectively.
\end{abstract}

Recently, ferroelectric multilayers and thin films with graded composition across the film to the substrate have been reported to exhibit striking properties that were not observed in conventional ferroelectric layers. The artificially enhanced chemical heterogeneity and the lattice strain introduced in these types of materials are attributed to their better properties than normal ferroelectric single layers. ${ }^{1-3}$ The (1 $-x) \mathrm{Pb}\left(\mathrm{Mg}_{1 / 3} \mathrm{Nb}_{2 / 3}\right) \mathrm{O}_{3}-x \mathrm{PbTiO}_{3} \quad(x=0,0.1,0.2,0.3), \quad$ also known as PMN-PT, is a well known relaxor ferroelectric which has been studied extensively with various compositions of PT and it has been proven as a promising material for solid state actuators and multilayer capacitors. ${ }^{4}$ The solid solution of 67\% PMN and 33\% PT is known as the morphotropic phase boundary composition in the PMN-PT phase diagram and is also found to have better properties than any other relaxor composition. ${ }^{5}$ The characteristic properties of the relaxor ferroelectrics are diffused phase transition with frequency dependent dielectric maxima, frequency independent behavior of dielectric constant well above the transition, slim loop hysteresis and a nonlinear dielectric response with respect to bias voltages. ${ }^{6,7}$

PMN-PT relaxors are an $\mathrm{ABO}_{3}$-type cubic perovskite with the presence of nanometer size polar clusters of $\mathrm{Mg}$ and $\mathrm{Nb}$ associated with a local charge imbalance and a 1:1 chemical ordering of B site within the polar cluster. The local symmetry of these polar clusters with rhombohedral symmetry different from the global nonpolar matrix with a cubic symmetry is a widely accepted feature. ${ }^{8,9}$ The presence of short-range interaction among these polar clusters was believed to be responsible for the diffusiveness in phase transition and also the existence of high spontaneous polarization values well above the transition temperature. ${ }^{10}$ This in turn gives rise to the enhancement of the properties dependent on $P_{s}$ even well above the transition temperature. ${ }^{6,7}$ The primary motive of this work is to study the variation of the physical properties, which depends on the microscopic fluctuations in the compositions, which is introduced by means of enhancing the chemical heterogeneity across the material. The PT

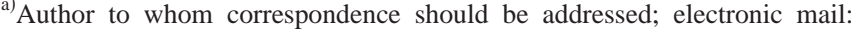
sbk@mrc.iisc.ernet.in
}

content was varied in different layers, which in turn introduces a huge spatial distribution of the density of the polar clusters and the lattice strain across the film. Whereas, the lattice strain is expected to be concentrated at the interfaces of individual layers and extend to few lattice planes in spite of the sharpness of the interfaces. Compositional heterogeneity along with the lattice strain may offer additional advantages in tuning the physical properties of the system. ${ }^{1-3}$

PMN-PT layers of different compositions were deposited using multi target pulsed laser ablation deposition. Four different targets of $(1-x) \mathrm{PMN}-x \mathrm{PT}$ were used, where $x$ takes the values of $0.3,0.2,0.1,0$ whereas the targets were prepared by the Columbite process and a $\mathrm{KrF}$ excimer laser $(248 \mathrm{~nm})$ was used for the ablation. ${ }^{11}$ The stacking sequences of the layers were as follows: $\mathrm{PMN} / 0.9 \mathrm{PMN}-$ 0.1PT / 0.8 PMN-0.2 PT / 0.7 PMN-0.3 PT / $\mathrm{La}_{0.5} \mathrm{Sr}_{0.5} \mathrm{CoO}_{3}$ (LSCO)/substrate. The usage of LSCO as a template layer has been established in the process of fabrication of PMN-PT layers on platinum coated silicon substrates. ${ }^{12,13}$ Each layer of different composition was deposited at $650^{\circ} \mathrm{C}$ for 5 minutes at an oxygen ambient pressure of 100 mTorr. The total thickness of the film was kept at around $450 \mathrm{~nm}$, with an individual layer thickness of around $120 \mathrm{~nm}$ measured by cross sectional view in scanning electron microscope. The crystallinity of the films was characterized by using $\mathrm{x}$-ray diffraction (XRD). The electrical characterization was carried out in a metal-insulator-metal configuration by depositing gold dots on top of the film by thermal evaporation, which acted as a top electrode, and the corner of platinum surface on the substrate was masked during deposition for bottom electrode. The top electrodes were annealed at $350{ }^{\circ} \mathrm{C}$ for $20 \mathrm{~min}$ for the adhesion of the same to the film. The dielectric phase transition behavior $(\varepsilon-T)$ and capacitance voltage $(C-V)$ characteristics were measured using an impedance analyzer of Agilent technologies model 4294A from $100 \mathrm{~Hz}$ to $1 \mathrm{MHz}$. The polarization hysteresis measurements were carried out using a RT66A setup operated in a Sawyer-Tower mode. ${ }^{14}$

The XRD pattern shown in Fig. 1. reveals a pyrochlore free, highly oriented film along (100) direction. The peaks were found to be broadened due to the internal stress present 


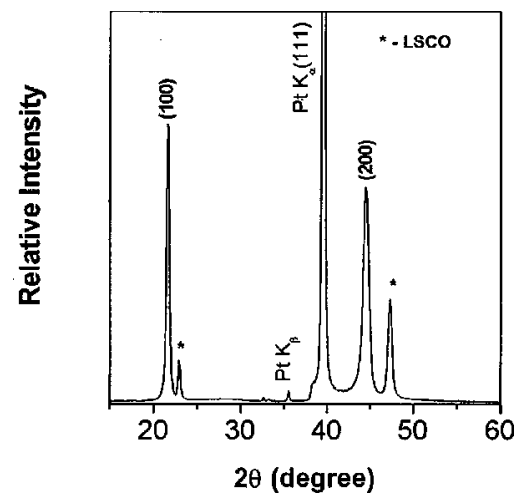

FIG. 1. XRD pattern of PMN-PT multilayer thin film.

in the system. This was confirmed from the excess broadening from that of the single layer XRD patterns whose grain structure and morphology were found to be the same. Figure 2 shows the temperature dependence of the dielectric permittivity, which was found to be an envelope of all the individual layer compositions. It can be visualized as the top pure PMN layer would undergo a transition followed by the consecutive layers and hence there will be individual layer dominance at the corresponding temperature regime and hence the enhancement in the diffusive nature of the phase transition. The phase transition was found to be broad and the dielectric constant was almost constant for a wide range of temperature for about $300-375 \mathrm{~K}$. The frequency independent behavior above the overall dielectric maximum temperature $\left(T_{m}\right)$ was also observed only at temperatures well above the $T_{m}$. This could be due to the individual layer's contribution having different $T_{m}$ when characterized individually. The enhanced diffusiveness could be because of the individual layer dominance at each temperature regime or an overall effect of the stress and chemical heterogeneity across the film.

The dielectric phase transition of these multilayers cannot be explained in terms of conventional relaxor ferroelectric system with single composition. Since the structure has a combination of different layers with different transition values, the dielectric properties are expected to have an overall effect on their temperature dependence. ${ }^{18}$ In principle the dielectric constant will be a combination of the following terms: (i) Dielectric constant of the individual layers with their own temperature dependence and relaxation time, (ii) the three active interfaces present between the relaxor layers and a passive interface between the LSCO and the bottom layer and (iii) the interaction of the two consecutive layers

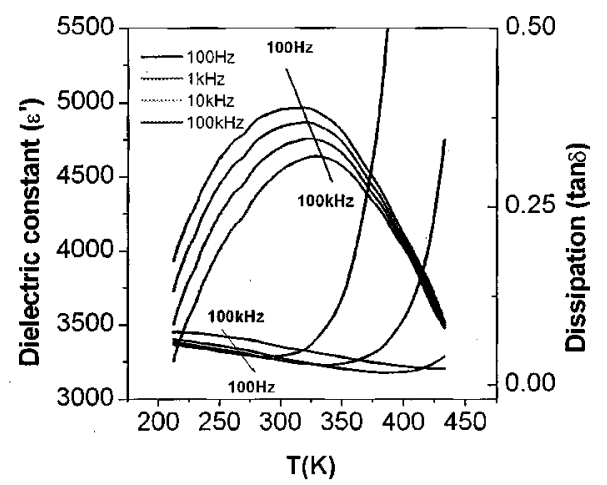

FIG. 2. Dielectric phase transition of PMN-PT multilayer thin films.

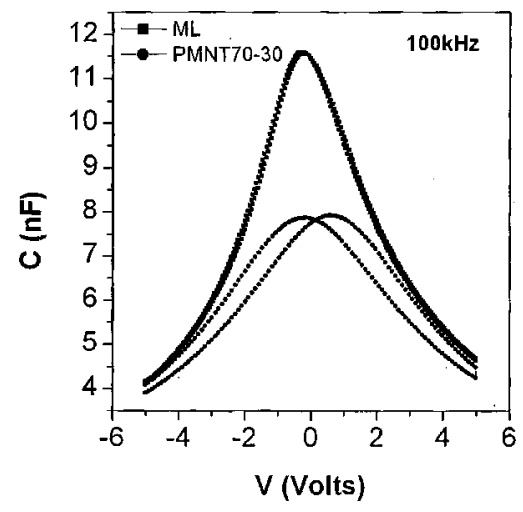

FIG. 3. Capacitance-voltage behavior of multilayer and PMN-PT 70-30 thin films.

though the interface by means of a coupling factor depending on the thickness of the individual layers, which in turn would modify the individual layer responses. A more detailed theoretical study involving the thickness of the individual layer and their polarization is required to understand the actual physical phenomena undergoing in the influence of the interface on the adjacent layers explained above. ${ }^{15}$ Hence, the enhanced diffusiveness could be due to the influence of individual layer composition at their corresponding temperature regime and the internally stressed regions due to the interfaces present across the film. The presence of the spatial variation of both the density and size of these polar clusters and the internal stress present at the interfaces play a major role in the enhancement of the diffusiveness of the dielectric phase transition. Moreover, a detailed thermodynamic free energy analysis including the strain and the spatial variation of polarization in microscopic scale is required to understand the collective physics involved in the phase transition. The loss factor $(D)$ was found to be 0.033 for $10 \mathrm{kHz}$ at room temperature $(303 \mathrm{~K})$ that was slightly higher than expected, but was lesser than the loss values reported for PMN-PT superlattices. ${ }^{16}$

The capacitance voltage $(C-V)$ behavior of these graded films is shown in Fig. 3. These compositionally varying multilayered films showed higher nonlinear dielectric response as a function of bias voltage and the high frequency $(100 \mathrm{kHz})$ tunability of the films was calculated to be around $70 \%-74 \%$ which is well above the tunability of any other single layer of same thickness $(50 \%-54 \%)$. The 70 PMN-30 PT, known to have better properties than any of the single layer composition taken for study, is used for com-

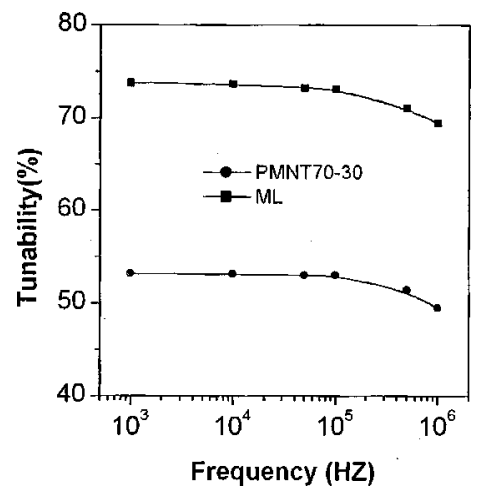

FIG. 4. Tunability of both multilayer and PMN-PT 70-30 thin films at different frequencies. 

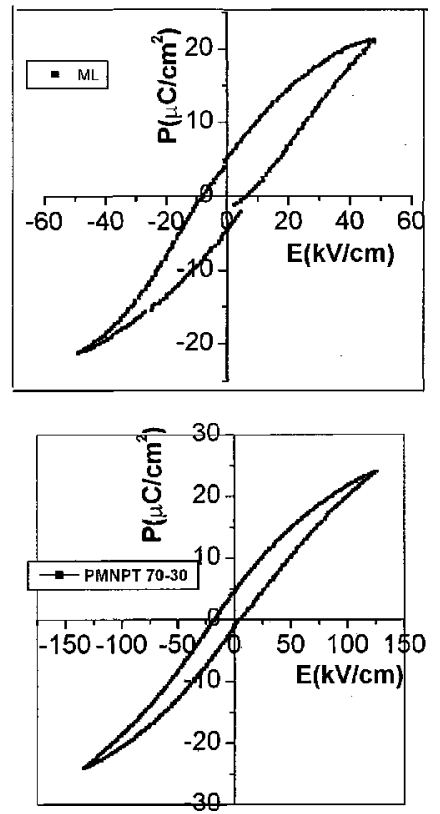

FIG. 5. (a) Polarization hysteresis of multilayered film. (b) Polarization hysteresis of PMN-PT 70-30 film.

parison and shown in the figure. The tunability was calculated using the equation given below ${ }^{17}$

$$
\text { Tunability }=\frac{\varepsilon(T, 0)-\varepsilon(\mathrm{T}, \mathrm{E})}{\varepsilon(T, 0)} \times 100 \% \ldots \ldots(a),
$$

where $\varepsilon(T, 0)$ is the dielectric permittivity at a given temperature and zero field, $\varepsilon(T, E)$ is the dielectric permittivity at the same temperature and at the maximum field. In our case the $C-V$ was measured at room temperature. Figure 4 shows the tunability of the multilayered film at various frequencies and the 70 PMN-30 PT single layer composition. The tunability of individual layers of a relaxor composition is generally explained in terms of the rotation of randomly oriented polar clusters, which acts like nano domains and their separation acting as a domain walls. The domain-type dynamics of these polar clusters is evident by means of considering an ergodic phase of a relaxor material above transition. ${ }^{18,19}$ The enhancement of tunability in the multilayers could be due to the stress present in the lattice and the distribution of nano polar regions across the film due to the variation of lead titanate. Though these films showed a tunability of around $74 \%$, they were found to be less than the well known high frequency tunable materials like $\mathrm{SrTiO}_{3} / \mathrm{BaTiO}_{3}$ superlattice structures by around $10 \%-15 \%$ in the measured frequency regime. ${ }^{2}$ Measurements are currently planned at higher frequencies. The $C-V$ curve at room temperature showed a very thin butterfly loop kind of behavior. Figure 5(a) shows the polarization hysteresis of a multilayered thin film and Fig. 5(b) shows the polarization of single layer with 70-30 composition; both the films showed a slim loop characteristic. The multilayered films showed a remnant polarization of around $6 \mu \mathrm{C} / \mathrm{cm}^{2}$.

Similar to capacitance-voltage behavior the $P-E$ curve will also be dominated by the rotation of the randomly ori- ented polar clusters present in the system. The asymmetry seen in the hysteresis between the first and the third quadrant may not be because of the asymmetric electrodes used on top and bottom, rather the system as a whole itself has an asymmetric band structure across the film due to the variation of composition near the two electrodes. Hence, this asymmetry in the band structure across the film could be attributed to the asymmetry observed in the hysteresis loop. ${ }^{20}$ The multilayered films showed a lower coercive field than that of the single layer with 70-30 compositions as observed in the $C$ - $V$ analysis. This reduction in coercive field could be attributed to the presence of both the ergodic and nonergodic phase at room temperatures. ${ }^{18}$

As the summary of the work we present that the variation or the enhancement of the chemical heterogeneity across the layer and the interfacial stress introduced due to a small variation in the lattice parameters of the each layer might correspond to the enhancement of the diffusiveness of the phase transition. Moreover, the phase transition is dominated by the individual layer response at corresponding temperature dominance. The tunability of these multilayers was found to be higher than any of the single layer compositions and was close to the tunability reported for superlattice structures of PMN-PT. ${ }^{16}$ This enhancement in tunability can also be attributed to the spatial fluctuation of composition and randomly oriented nano polar clusters distributed across the film. These mulitlayers exhibited a slim polarization loop characteristic, which is a typical behavior of any relaxor material.

${ }^{1}$ J. V. Mantese, N. W. Schubring, A. L. Mchelli, A. B. Catalang, M. S. Mohammed, R. Naik, and G. W. Avner, Appl. Phys. Lett. 71, 2047 (1997).

${ }^{2}$ J. Kim, Y. Kim, Y. S. Kim, J. Lee, L. Kim, and D. Jung, Appl. Phys. Lett. 80, 3581 (2002).

${ }^{3}$ K. O. Shimoyama, M. Kiyohara, K. Kubo, A. Uedona, and K. Yamabe, J. Appl. Phys. 92, 4625 (2002).

${ }^{4}$ K. Uchino, Piezoelectric Actuators and Ultrasonic Motors (Kluwer, Dordrecht (1996), and references therein.

${ }^{5}$ T. R. Shrout and J. F. Fielding Jr., IEEE Ultrasonic Symposium, 1990, p. 711.

${ }^{6}$ L. E. Cross, Ferroelectrics 151, 305 (1987).

${ }^{7}$ G. A. Smolensky and A. I. Agranovskaya, Sov. Phys. Solid State 1, 1429 (1959).

${ }^{8}$ E. Husson, M. Chubb, and A. Morell, Mater. Res. Bull. 23, 357 (1988).

${ }^{9}$ P. K. Davies and M. A. Akbas, J. Phys. Chem. Solids 61, 159 (2000).

${ }^{10}$ B. E. Vugmeister and H. Rabitz, Phys. Rev. B 57, 7581 (1998).

${ }^{11}$ S. Swartz and T. Shrout, Mater. Res. Bull. 17, 1245 (1982).

${ }^{12}$ A. Laha, P. Victor, and S. B. Krupanidhi, Integr. Ferroelectr. 46, 143 (2002).

${ }^{13}$ A. Laha, S. Saha, and S. B. Krupanidhi, Thin Solid Films 424, 274 (2003).

${ }^{14}$ C. B. Sawyer and C. H. Tower, Phys. Rev. 35, 269 (1930).

${ }^{15}$ K. H. Chew, L. H. Ong, J. Osman, and D. R. Tilley, Appl. Phys. Lett. 77, 2755 (2000).

${ }^{16}$ Y. Lu, Appl. Phys. Lett. 85, 979 (2004).

${ }^{17}$ S. G. Lu, X. H. Zhu, C. L. Mak, K. H. Wong, H. L.W. Chan, and C. L. Choy, Appl. Phys. Lett. 82, 2877 (2003).

${ }^{18}$ A. E. Glazounov, A. K. Taganstev, and A. J. Bell, Phys. Rev. B 53, 11281 (1996).

${ }^{19}$ M. Abplanalp, D. Barosova, P. Bridenbaugh, J. Erhart, J. Fousek, P. Gunter, J. Nosek, and M. Sulc, J. Appl. Phys. 91, 3797 (2002).

${ }^{20}$ S. J. Lee, S. E. Moon, H. C. Ryu, M. H. Kwak, Y. T. Kim, and S. K. Han, Appl. Phys. Lett. 82, 2133 (2003). 